\title{
Caracterización sociodemográfica y clínica de pacientes con dolor crónico de espalda, Cienfuegos 2019
}

\author{
Sociodemographic and clinical characterization in patients \\ with chronic back pain, Cienfuegos 2019
}

\author{
A. J. Pomares Avalos', D. F. Zaldivar Pérez² y M. A. Vázquez Núñez
}

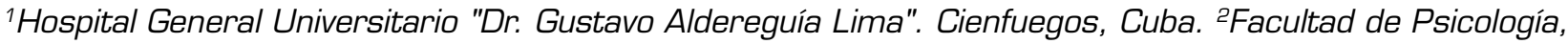
Universidad de La Habana. Cuba. ${ }^{3}$ Universidad de Ciencias Médicas. Cienfuegos, Cuba

\section{ABSTRACT}

Objective: To determine the main sociodemographic and clinical characteristics present in patients with chronic back pain of the population in Cienfuegos.

Material and Method: A descriptive study was carried out, as a series of cases, in the months from May to October 2019, at the Dr. Gustavo Aldereguía Lima Hospital in the province of Cienfuegos, Cuba. A causal non probabilistic sampling was performed or to a convenience of voluntary participants. The sample was made up of a total of 70 patients with diagnosis of chronic back pain, initially valued by orthopedic specialization and Physical Rehabilitations, greater than 20 years of age, without cognitive impairment, nor severe psychic disease, which gave their informed consent to participate in the study. The sociodemographic variables were studied: age, sex, school level, occupation and clinical variables: etiological diagnosis, pain intensity, frequency of appearance, time of evolution, type of treatment for relief and disability. The instruments used were: Semi-structured Interview, Visual Analog Scale (VAS) and Oswestry Low Back Pain Disability Scale. Statistical analysis was performed in the database of the SPSS statistical package.

Results: patients aged between 41 and 60 years (60.2\%), female (74.3\%), with a higher level [38.6\%) and occupations involving frequent physical exertion prevailed (61, $4 \%$ ). Nonspecific etiological diagnosis (62.8\%), severe pain intensity (55.7\%), stable frequency $[55.7 \%$ ) with more than 5 years of disease

Pomares Avalos AJ, Zaldívar Pérez DF, Vázque Núñez MA. Caracterización sociodemográfica y clínica de pacientes con dolor crónico de espalda, Cienfuegos 2019. Rev Soc Esp Dolor. 2020;27(4):239-245

\section{RESUMEN}

Objetivo: Determinar las principales características sociodemográficas y clínicas presentes en pacientes con dolor crónico de espalda de la población cienfueguera.

Material y método: Se realizó un estudio descriptivo, de serie de casos, en los meses comprendidos de mayo a octubre de 2019, en el Hospital "Dr. Gustavo Aldereguía Lima", de la provincia de Cienfuegos, Cuba. Se realizó un muestreo no probabilístico casual o a conveniencia de participantes voluntarios. La muestra quedó conformada por un total de 70 pacientes con diagnóstico de dolor crónico de la espalda, valorados inicialmente por especialistas de Ortopedia o Medicina Física y Rehabilitación, mayores de 20 años de edad, sin deterioro cognitivo, ni enfermedad psíquica severa, que dieron su consentimiento informado para participar en el estudio.

Se estudiaron las variables sociodemográficas: edad, sexo, nivel escolar, ocupación y las variables clínicas: diagnóstico etiológico, intensidad del dolor, frecuencia de aparición, tiempo de evolución, tipo de tratamiento para su alivio y discapacidad. Los instrumentos empleados fueron: entrevista semiestructurada, escala visual analógica (EVA) y escala de incapacidad por dolor lumbar de Oswestry. El análisis estadístico se realizó en la base de datos del paquete estadístico SPSS.

Resultados: Prevalecieron los pacientes del grupo de edad de 41 a 60 años (60,2\%), del sexo femenino [74,3\%), con nivel superior $[38,6 \%$ ) y las ocupaciones que conllevan esfuerzo físico frecuente $[61,4 \%]$.

Recibido: 02-03-2020

Aceptado: 06-05-2020

Correspondencia: Ahmed José Pomares Avalos ahmed.pomares@gal.sld.cu 
progression (48.6\%), predominated. It also highlights medical treatment (85.7\%) and moderate disability [54.3\%].

Conclusions: people at work-related ages are subject to a series of physical factors, typical of the activities of daily life that, in addition, are nuanced by a gender approach that derives from the culture and socialization process.

Key words: Chronic pain, back pain.
Predominó el diagnóstico etiológico inespecífico [62,8\%), la intensidad del dolor severa $(55,7 \%)$, de frecuencia estable $[55,7 \%$ y más de 5 años de evolución de la enfermedad (48,6\%). Se destaca además el tratamiento médico $[85,7 \%$ y la discapacidad moderada [54,3 \%].

Conclusiones: Las personas en edades laboralmente activas se encuentran sujetos a una serie de factores físicos, propios de las actividades de la vida cotidiana que por demás están matizadas por un enfoque de género que se deriva de la cultura y el proceso de socialización.

Palabras clave: Dolor crónico, dolor de espalda.

\section{INTRODUCCIÓN}

El dolor crónico de espalda es un dolor que se origina en los músculos, nervios, huesos, articulaciones u otras estructuras de la columna vertebral, que abarca desde la zona cervical hasta la zona del cóccix, con una duración de al menos 3 meses (1).

Constituye una de las manifestaciones más frecuentes de los síndromes dolorosos, con una etiología multifacética, donde no solo interactúan variables biomédicas, sino también influye la cultura, las creencias, los estados de ánimo y la capacidad para hacerle frente (2).

Múltiples estudios coinciden en que esta patología afecta un segmento importante de la población; así, por ejemplo, Europa reporta cifras del $9 \%$, Alemania reporta una prevalencia entre el 10 y el $27 \%$, mientras que en el Reino Unido constituye la principal causa de discapacidad y carga financiera para la salud y la sociedad [3-5].

Su repercusión supera el ámbito de la salud para convertirse una carga socioeconómica, dada la alta incidencia en la población laboralmente activa. Los gastos económicos por conceptos de ausencia laboral, certificados y visitas médicas, ascienden a cifras alarmantes $(6,7)$.

Cuba no cuenta con registros estadísticos o documentos oficiales que reflejen la cifra real de pacientes con dolor crónico de espalda, ni su distribución sociodemográficas en la población, lo que limita en muchas ocasiones las investigaciones sobre el tema y las acciones encaminadas a mejorar esta situación.

La provincia de Cienfuegos, por su parte, presenta la misma situación en cuanto a la carencia de documentación oficial. Sin embargo, desde el año 2016 se han desarrollado una serie de estudios dirigidos a estos pacientes, que reflejan la problemática de salud que representa este padecimiento para el territorio cienfueguero $[8,9]$.

El conocimiento de las características clínicas del dolor y su distribución sociodemográfica son herramientas de gran valor terapéutico, por lo que, se decide realizar un estudio con el objetivo de determinar las principales características sociodemográficas y clínicas presentes en los pacientes con dolor crónico de espalda en la población cienfueguera.

\section{METODOLOGÍA}

Tipo de estudio y contexto: se realizó un estudio descriptivo, de serie de casos, en el Hospital Dr. Gustavo Aldereguía Lima, de la provincia de Cienfuegos, Cuba, en los meses comprendidos de mayo a octubre de 2019.

Muestra: se realizó un muestreo no probabilístico casual o a conveniencia de participantes voluntarios, con los pacientes que fueron atendido en consulta de Ortopedia durante el periodo de investigación declarado. Se utilizó este tipo de muestreo con el objetivo de acceder a la mayor cantidad de sujetos posibles y por no contarse con un registro estadístico que permitiera estadificar este grupo de pacientes. La muestra quedó conformada por un total de 70 personas que cumplieron los siguientes criterios de inclusión: paciente con diagnóstico de dolor crónico de la espalda, valorados inicialmente por especialistas de Ortopedia o Medicina Física y Rehabilitación, mayores de 20 años de edad, sin deterioro cognitivo ni enfermedad psíquica severa, que dieron su consentimiento informado para participar en el estudio.

Variables de estudio: se estudiaron las variables sociodemográficas: edad (20-40, 41-60 y más de 60 años), sexo (masculino y femenino), nivel escolar [6. ${ }^{\circ}, 9^{\circ}, 12 .^{\circ}$, Técnico medio y Nivel superior], ocupación (con esfuerzo físico frecuente y sin esfuerzo físico frecuente] y las variables clínicas: diagnóstico etiológico (fibromialgia, patología de disco, traumatismos, osteoporosis, artritis, compresión de vértebras y causa inespecífica), intensidad del dolor (0-3 dolor leve, 4-7 dolor moderado y 8-10 dolor severo], frecuencia de aparición (recurrente y estable), tiempo de evolución ( 6 meses a 1 año, 1 año a 5 años y más de 5 años), tipo de tratamiento para su alivio (médico, psicoterapéutico, ambos y ninguno] y discapacidad funcional (incapacidad leve 0-20, moderada 21-60 y grave más de 60).

Instrumentos en la recogida de la información: se empleó la entrevista semiestructurada con el objetivo de recoger datos sociodemográficos y de interés clínico como: la frecuencia de aparición del dolor, los tratamientos utilizados para el alivio y el tiempo de evolución del dolor, además se utilizó la Escala Visual Analógica [EVA] y la Escala de Incapacidad por dolor lumbar de Oswestry, la cual fue validada en la población cienfue- 
guera con motivo de la presente investigación (10). Los instrumentos se aplicaron bajo condiciones adecuadas en una sesión de trabajo de forma individual a cada paciente.

Métodos estadísticos: el análisis estadístico de la información se realizó en la base de datos del paquete estadístico SPSS. Se utilizó la frecuencia y el porcentaje.

Aspectos éticos de la investigación: se tuvo en cuenta los aspectos éticos y jurídicos en la obtención de la información. Se utilizó el consentimiento informado de los pacientes y de la institución para la realización de la investigación y para la consiguiente aplicación de los instrumentos seleccionados para estos fines.

\section{RESULTADOS}

La Tabla I muestra la caracterización sociodemográfica de los pacientes de estudio. Predominaron los pacientes en el rango de edad entre 41 y 60 años [60,2 \%), seguido de más de 60 años (28,6 \%). En cuanto al sexo, prevaleció el femenino sobre el masculino (74,3 \%, 25,7\%, respectivamente). Prevalecieron los pacientes con escolaridad superior $[38,6 \%$ y las ocupaciones que conllevan esfuerzo físico frecuente [61,4\%].

La Tabla II muestra un predominio de pacientes con diagnóstico etiológico inespecífico (45,7\%), seguido de las patologías discales [22,9\%). La mayor parte de los pacientes tienen una percepción del dolor severa (55,7\%); le sigue de la moderada (35,7\%). La frecuencia de aparición del dolor que predominó fue la estable

TABLA I

CARACTERIZACIÓN DE LOS PACIENTES

DEL ESTUDIO EN CUANTO A LAS VARIABLES SOCIODEMOGRÁFICAS

\begin{tabular}{|c|c|c|c|}
\hline \multirow{4}{*}{ Edad } & $20-40$ & No. & $\%$ \\
\cline { 2 - 4 } & $41-60$ & 8 & 11,4 \\
\cline { 2 - 4 } & +60 & 20 & 60,0 \\
\hline \multirow{3}{*}{ Sexo } & Masculino & 18 & 25,6 \\
\cline { 2 - 4 } & Femenino & 52 & 74,3 \\
\hline \multirow{4}{*}{ Escolaridad } & $6^{\circ}$ & 4 & 5,7 \\
\cline { 2 - 4 } & $9^{\circ}$ & 2 & 2,9 \\
\cline { 2 - 4 } & 12 & 17 & 24,3 \\
\cline { 2 - 4 } & TM & 20 & 28,6 \\
\hline \multirow{3}{*}{ Ocupación } & $\begin{array}{c}\text { Universitario } \\
\text { Con esfuerzo físico } \\
\text { frecuente }\end{array}$ & 27 & 38,6 \\
\cline { 2 - 4 } & $\begin{array}{c}\text { Sin esfuerzo físico } \\
\text { frecuente }\end{array}$ & 27 & 38,4 \\
\hline
\end{tabular}

TABLA II

DISTRIBUCIÓN DE LOS PACIENTES DEL ESTUDIO

EN CUANTO A LAS VARIABLES CLÍNICAS Y TIPO DE TRATAMIENTO UTILIZADO

\begin{tabular}{|c|c|c|c|}
\hline \multicolumn{2}{|c|}{ Variables } & No. & $\%$ \\
\hline \multirow{7}{*}{$\begin{array}{l}\text { Diagnóstico } \\
\text { etiológico }\end{array}$} & Fibromialgia & 5 & 7,1 \\
\hline & Osteoporosis & 3 & 4,3 \\
\hline & Artritis & 6 & 8,6 \\
\hline & Traumatismo & 7 & 10,0 \\
\hline & $\begin{array}{l}\text { Compresión } \\
\text { de vértebras }\end{array}$ & 1 & 1,4 \\
\hline & Patología discal & 16 & 22,9 \\
\hline & Inespecífico & 32 & 45,7 \\
\hline \multirow{3}{*}{$\begin{array}{l}\text { Intensidad del } \\
\text { dolor }\end{array}$} & Leve & 6 & 8,6 \\
\hline & Moderada & 25 & 35,7 \\
\hline & Severa & 39 & 55,7 \\
\hline \multirow{2}{*}{$\begin{array}{l}\text { Frecuencia de } \\
\text { aparición del } \\
\text { dolor }\end{array}$} & Recurrente & 31 & 44,3 \\
\hline & Estable & 39 & 55,7 \\
\hline \multirow{3}{*}{$\begin{array}{l}\text { Tiempo de } \\
\text { evolución del } \\
\text { dolor }\end{array}$} & 6 meses-1 año & 7 & 10,0 \\
\hline & 1 año-5 año & 29 & 41,4 \\
\hline & + 5 años & 34 & 48,6 \\
\hline \multirow{4}{*}{$\begin{array}{l}\text { Tipo de } \\
\text { tratamiento } \\
\text { para el alivio del } \\
\text { dolor }\end{array}$} & Médico & 60 & 85,7 \\
\hline & Psicoterapéutico & 1 & 1,4 \\
\hline & Ambos & 9 & 12,9 \\
\hline & Ninguno & - & - \\
\hline \multirow{3}{*}{$\begin{array}{l}\text { Discapacidad } \\
\text { funcional }\end{array}$} & Leve & 16 & 22,9 \\
\hline & Moderada & 38 & 54,3 \\
\hline & Grave & 16 & 22,9 \\
\hline
\end{tabular}

[55,7\%] y los pacientes con más de 5 años de evolución de la enfermedad (48,6\%). Obsérvese además el predominio del tratamiento médico $(85,7 \%)$. Llama la atención que solo el 12,9\% ha recibido tratamiento multidisciplinario. Por último, predominó el grado de discapacidad moderado (54,3\%).

En la Tabla III se observa que las actividades de la vida cotidiana donde mayor discapacidad existe son en levantar pesos (98,6\%), estar de pie $(97,1 \%$ ) y estar sentado $[94,3 \%)$.

En la Tabla IV se observa un predominio de la intensidad del dolor severa en todos los grupos de edades. Es necesario destacar que el grupo de edad donde mayor cantidad de pacientes existe con una intensidad del dolor severa fueron los de 41-60 años, [56,4 \%). 
Con respecto al sexo y a las diferentes categorías de la intensidad del dolor, el $50 \%$ de los pacientes masculinos perciben una intensidad severa, mientras que en el caso de los femeninos es el 57,7\%. Un dato interesante resulta que de los 39 pacientes que perciben intensamente el dolor, 30 (76,9\%) son del sexo femenino mientras que solo $9(23,1 \%)$, representan al sexo masculino.

\section{TABLA III}

GRADO DE DISCAPACIDAD FUNCIONAL EN DIFERENTES ACTIVIDADES DE LA VIDA COTIDIANA

\begin{tabular}{|l|c|c|}
\hline \multicolumn{1}{|c|}{ Variable } & No. & $\%$ \\
\hline Intensidad del dolor & 67 & 95,7 \\
\hline Cuidados personales & 53 & 75,7 \\
\hline Levantar peso & 69 & 98,6 \\
\hline Andar & 44 & 62,9 \\
\hline Estar sentado & 66 & 94,3 \\
\hline Estar pie & 68 & 97,1 \\
\hline Dormir & 31 & 44,3 \\
\hline Actividad sexual & 55 & 78,6 \\
\hline Vida social & 59 & 84,3 \\
\hline Viajar & 61 & 87,1 \\
\hline
\end{tabular}

En la Tabla $V$ se obtiene que en los pacientes entre 20-40 años predomina la discapacidad en niveles moderados y graves, ambas con el 37,5\%). Datos similares se obtienen en los pacientes mayores de 60 años, donde tanto la discapacidad moderada como la grave coinciden $[45,0 \%)$. Sin embargo, en los pacientes en edades comprendidas entre 41 y 60 años, la discapacidad prevalece en niveles moderados [61,9\%). Un dato significativo resulta que de los 16 pacientes con discapacidad grave, el mayor grupo [56,3 \%] son mayores de 60 años. Con respecto al sexo, se obtiene que en cuanto al sexo masculino el mayor número de pacientes presenta una discapacidad grave $[38,9 \%$, no así el sexo femenino, donde predomina la discapacidad moderada (63,5\%). Sin embargo, los resultados arrojan que existen más mujeres que hombres con un grado de discapacidad grave $[56,2 \%$ y $43,8 \%$, respectivamente).

La Tabla VI refleja que los pacientes que perciben la intensidad del dolor leve presentan a la vez discapacidad leve, mientras que los pacientes que perciben la intensidad del dolor moderada e intensa presentan una discapacidad moderada. Por otro parte, los pacientes con un grado de discapacidad leve perciben el dolor con moderada intensidad, mientras aquellos pacientes con moderada y grave discapacidad perciben intensamente el dolor.

La Tabla VII muestra un predomino de las patologías en aquellas ocupaciones que realizan esfuerzo físico frecuente; sin embargo, resulta llamativo que tanto la fibromialgia como las patologías de disco prevalecen en personas que no refieren sobrecarga física.

TABLA IV

RELACIÓN ENTRE LA INTENSIDAD DEL DOLOR CON LA EDAD Y EL SEXO

\begin{tabular}{|c|c|c|c|c|c|}
\hline \multirow{2}{*}{\multicolumn{2}{|c|}{ Variables }} & \multicolumn{3}{|c|}{ Intensidad del dolor } & \multirow{3}{*}{$\begin{array}{c}\text { Total } \\
8 \\
100 \%\end{array}$} \\
\hline & & \multirow{2}{*}{$\begin{array}{c}\text { Leve } \\
0 \\
0,0 \%\end{array}$} & \multirow{2}{*}{$\begin{array}{c}\text { Moderada } \\
37,5 \%\end{array}$} & \multirow{2}{*}{$\begin{array}{c}\text { Severa } \\
5 \\
62,5 \%\end{array}$} & \\
\hline \multirow{3}{*}{ Edad } & $20-40$ & & & & \\
\hline & $41-60$ & $\begin{array}{c}4 \\
9,5 \%\end{array}$ & $\begin{array}{c}16 \\
38,1 \%\end{array}$ & $\begin{array}{c}22 \\
52,4 \%\end{array}$ & $\begin{array}{c}42 \\
100 \%\end{array}$ \\
\hline & +60 & $\begin{array}{c}2 \\
10,0 \%\end{array}$ & $\begin{array}{c}6 \\
30,0 \%\end{array}$ & $\begin{array}{c}12 \\
60,0 \%\end{array}$ & $\begin{array}{c}20 \\
100 \%\end{array}$ \\
\hline \multicolumn{2}{|l|}{ Total } & $\begin{array}{c}6 \\
8,6 \%\end{array}$ & $\begin{array}{c}25 \\
35,7 \%\end{array}$ & $\begin{array}{c}39 \\
55,7 \%\end{array}$ & $\begin{array}{c}70 \\
100 \%\end{array}$ \\
\hline \multirow{2}{*}{ Sexo } & Masculino & $\begin{array}{c}1 \\
5,6 \%\end{array}$ & $\begin{array}{c}8 \\
44,4 \%\end{array}$ & $\begin{array}{c}9 \\
50,0 \%\end{array}$ & $\begin{array}{c}18 \\
100 \%\end{array}$ \\
\hline & Femenino & $\begin{array}{c}5 \\
9,6 \%\end{array}$ & $\begin{array}{c}17 \\
32,7 \%\end{array}$ & $\begin{array}{c}30 \\
57,7 \%\end{array}$ & $\begin{array}{c}52 \\
100 \%\end{array}$ \\
\hline \multicolumn{2}{|l|}{ Total } & $\begin{array}{c}6 \\
8,6 \%\end{array}$ & $\begin{array}{c}25 \\
35,7 \%\end{array}$ & $\begin{array}{c}39 \\
55,7 \%\end{array}$ & $\begin{array}{c}70 \\
100 \%\end{array}$ \\
\hline
\end{tabular}


TABLA V

RELACIÓN ENTRE EL GRADO DISCAPACIDAD FUNCIONAL CON LA EDAD Y EL SEXO

\begin{tabular}{|c|c|c|c|c|c|}
\hline \multirow{2}{*}{\multicolumn{2}{|c|}{ Variable }} & \multicolumn{3}{|c|}{ Discapacidad } & \multirow{3}{*}{$\begin{array}{c}\text { Total } \\
8 \\
100 \%\end{array}$} \\
\hline & & \multirow{2}{*}{$\begin{array}{c}\text { Leve } \\
2 \\
25,0 \%\end{array}$} & \multirow{2}{*}{$\begin{array}{c}\text { Moderada } \\
3 \\
37,5 \%\end{array}$} & \multirow{2}{*}{$\begin{array}{c}\text { Grave } \\
3 \\
37,5 \%\end{array}$} & \\
\hline \multirow{3}{*}{ Edad } & $20-40$ & & & & \\
\hline & $41-60$ & $\begin{array}{c}12 \\
28,6 \%\end{array}$ & $\begin{array}{c}26 \\
61,9 \%\end{array}$ & $\begin{array}{c}4 \\
9,5 \%\end{array}$ & $\begin{array}{c}42 \\
100 \%\end{array}$ \\
\hline & +60 & $\begin{array}{c}2 \\
10,0 \%\end{array}$ & $\begin{array}{c}9 \\
45,0 \% \\
\end{array}$ & $\begin{array}{c}9 \\
45,0 \%\end{array}$ & $\begin{array}{c}20 \\
100 \%\end{array}$ \\
\hline \multicolumn{2}{|l|}{ Total } & $\begin{array}{c}16 \\
22,9 \% \\
\end{array}$ & $\begin{array}{c}38 \\
54,3 \% \\
\end{array}$ & $\begin{array}{c}16 \\
22,9 \% \\
\end{array}$ & $\begin{array}{c}70 \\
100 \% \\
\end{array}$ \\
\hline \multirow[b]{2}{*}{ Sexo } & Masculino & $\begin{array}{c}6 \\
33,3 \% \\
\end{array}$ & $\begin{array}{c}5 \\
27,8 \% \\
\end{array}$ & $\begin{array}{c}7 \\
38,9 \% \\
\end{array}$ & $\begin{array}{c}18 \\
100 \%\end{array}$ \\
\hline & Femenino & $\begin{array}{c}10 \\
19,2 \%\end{array}$ & $\begin{array}{c}33 \\
63,5 \% \\
\end{array}$ & $\begin{array}{c}9 \\
17,3 \%\end{array}$ & $\begin{array}{c}52 \\
100 \% \\
\end{array}$ \\
\hline \multicolumn{2}{|l|}{ Total } & $\begin{array}{c}16 \\
22,9 \% \\
\end{array}$ & $\begin{array}{c}38 \\
54,3 \% \\
\end{array}$ & $\begin{array}{c}16 \\
22,9 \% \\
\end{array}$ & $\begin{array}{c}70 \\
100 \% \\
\end{array}$ \\
\hline
\end{tabular}

TABLA VI

RELACIÓN ENTRE LA INTENSIDAD DEL DOLOR Y EL GRADO DE DISCAPACIDAD FUNCIONAL

\begin{tabular}{|l|l|c|c|c|c|}
\hline \multicolumn{2}{|c|}{ Variables } & \multicolumn{3}{c|}{ Discapacidad } & \multirow{2}{*}{ Total } \\
\cline { 3 - 6 } & \multirow{4}{*}{ Leve } & Leve & Moderada & Grave & \\
\cline { 2 - 5 } & & 3 & 2 & 1 & 6 \\
\cline { 2 - 5 } & \multirow{3}{*}{ Modensidad del dolor } & $50,0 \%$ & $33,3 \%$ & $16,1 \%$ & $100 \%$ \\
\cline { 2 - 6 } & \multirow{2}{*}{ Severa } & $36 \%$ & 12 & 4 & 25 \\
& & 4 & $24 \%$ & $16,0 \%$ & $100 \%$ \\
\hline \multirow{2}{*}{ Total } & $10,2 \%$ & $61,5 \%$ & $28,2 \%$ & $100 \%$ \\
\hline
\end{tabular}

TABLA VII

RELACIÓN ENTRE LA OCUPACIÓN Y EL DIAGNÓSTICO ETIOLÓGICO

\begin{tabular}{|l|c|c|c|}
\hline \multirow{2}{*}{ Diagnóstico etiológico } & \multicolumn{2}{|c|}{ Ocupación } & \multirow{2}{*}{ Total } \\
\cline { 2 - 3 } & $\begin{array}{c}\text { Con esfuerzo } \\
\text { fisico frecuente }\end{array}$ & $\begin{array}{c}\text { Sin esfuerzo } \\
\text { fisico frecuente }\end{array}$ & 5 \\
\hline Fibromialgia & 2 & 3 & 16 \\
\hline Patología de disco & 7 & 9 & 7 \\
\hline Traumatismos & 5 & 2 & 3 \\
\hline Osteoporosis & 3 & 0 & 6 \\
\hline Artritis & 3 & 3 & 1 \\
\hline Compresión de vértebras & 1 & 0 & 32 \\
\hline Causa inespecífica & 22 & 10 & 70 \\
\hline Total & 43 & 27 & \\
\hline
\end{tabular}




\section{ANÁLISIS Y DISCUSIÓN}

En la presente investigación prevalecen los pacientes en el rango de 40 a 60 años de edad, coincidiendo con otras investigaciones realizadas en el territorio $[8,9]$; no así con investigaciones internacionales que asocian esta patología al envejecimiento y a los procesos degenerativos propios de la tercera edad (11).

Sin embargo, este grupo de edad se encuentra laboralmente activo, lo que aumenta el riesgo de accidentes laborales, sobreesfuerzo físico y posturas inadecuadas, factores ampliamente relacionados con la experiencia de dolor (12).

Predominó el sexo femenino, lo que se encuentra en correspondencia con diversas investigaciones que plantean que, a pesar de que aún no es clara la prevalencia del dolor en relación al sexo, existen diferencias fisiológicas, anatómicas, neurales, hormonales, psicológicas y socioculturales que concluyen que la mujer reporta con mayor frecuencia el dolor y presenta un umbral más bajo respecto a los hombres $(13,14)$.

Otros estudios se refieren al papel de las variables socioculturales en el proceso salud y enfermedad, destacando que las cargas sociales, la doble jornada laboral tanto en su desempeño profesional como todas las actividades que garantizan el bienestar y la sobrevivencia de los individuos que comparten el hogar, los roles asumidos y el estatus que desempeña una mujer en la actualidad, guardan relación con las patologías que padece [15].

En relación con el nivel escolar predominaron los universitarios, datos que no se corresponden con otros estudios que han obtenido bajos niveles de escolaridad en este tipo de pacientes, incluso llegando a considerarlo como un factor de riesgo para la aparición desencadenamiento o empeoramiento del dolor (16).

Un alto grado de escolaridad puede optimizar las herramientas de búsqueda de información, favoreciendo el autocuidado y las conductas protectoras, propiciando el empleo de estrategias de afrontamiento más eficaces y adaptativas.

La mayoría de los pacientes del estudio presentan una ocupación laborar que demanda de esfuerzo físico, lo que favorece la aparición del dolor y la discapacidad. Diversos factores físicos contribuyen al dolor, como el levantamiento de pesos, las cargas físicas elevadas y el estrés postural. Este último ha sido considerado el más implicado, ya que incluye movimientos, como inclinarse y girar, que pueden ser frecuentes y repetidos, los cuales pueden conducir a degeneración discal y protrusiones discales de diversa magnitud $(12,16)$.

Se encontró un predominio del dolor crónico de espalda de causa inespecífica, resultado que coincide con otros autores que lo ubican en un rango entre 70 y el $85 \%$ (17).

Este diagnóstico etiológico es precisamente el más complejo y el de mayor relación con factores psicosociales, representando una de las causas más comunes de discapacidad y deterioro de la calidad de vida. Su naturaleza imprecisa y la ausencia de un daño tisular bien identificado complica el accionar terapéutico.

Se obtiene una intensidad del dolor severa, lo que coincide con un estudio realizado en Colombia, donde el $82 \%$ de los pacientes presentaron dolor de moderado a severo [17]. Otro estudio "Survey of Chronic Pain in Europe: Prevalence, Impacton Daily Life and Treatment", encuesta de gran relevancia mundial, demostró que el
$19 \%$ de los encuestados sufrían dolor crónico con una intensidad de moderado a severo (18). Asimismo, estos resultados coinciden con los estudios anteriores realizados en el territorio cienfueguero $[8,9]$.

Se obtiene además una intensidad del dolor severa en todos los grupos de edades. Mientras que algunos autores indican que en la medida que se envejece se experimentan cambios en la percepción de dolor, llegando a disminuir notablemente con el tiempo [19], otros autores como Fordyce argumentan que los pacientes añosos se quejan más de dolor que los jóvenes (20).

Otro dato interesante indica que el sexo femenino percibe mayor dolor que el masculino. Este resultado puede estar avalado porque las identidades masculinas son más represoras de emociones en comparación con las mujeres, y estas diferencias de género se explican debido a la influencia de una educación diferencial entre ambos sexos, donde la sociedad refuerza la libre expresión del dolor en la niña, pero no en el niño.

La intensidad del dolor es una característica clínica que genera mayor discapacidad y contribuye a la cronicidad del mismo, a la vez que se relaciona a una mayor presencia de emociones negativas [21].

Lo anterior se corrobora con el hecho de que los pacientes del estudio sufren el dolor hace más de 5 años y este se presenta de manera estable. Elementos que aportan a la discapacidad.

Es necesario destacar entonces que el dolor prolongado en el tiempo provoca una serie de eventos somáticos y psíquicos que conforman una cadena de factores que pueden tornarlo más complejo en cuanto a su estudio y terapéutica a implementar.

Con respecto a los tratamientos aplicados para el alivio del dolor, se observa un predominio del tratamiento médico exclusivo, coincidiendo con la mayoría de los estudios sobre el tema [22].

A pesar de la obsolescencia del paradigma biomédico clásico y del cambio hacia una concepción biopsicosocial en la interpretación y manejo del dolor, no existe todavía plena concienciación por parte del personal de salud acerca del abordaje integral del dolor, ya que las cifras de pacientes que son tratados exclusivamente con terapias médicas superan con creces a aquellos que son tratado multidisciplinariamente.

Las evidencias reflejan cómo los pacientes tratados conjuntamente con ambas técnicas (médicas y psicológicas] muestran una mayor reducción del dolor, de la incapacidad y de los estados emocionales negativos [2२].

En cuanto a la discapacidad funcional, prevalecieron las categorías moderada y grave, datos que se corresponden teniendo en cuenta la intensidad del dolor y el tiempo de evolución de la enfermedad referido por los pacientes de estudio. La literatura científica define la discapacidad como una consecuencia negativa del dolor crónico, resaltando que precisamente el carácter crónico de la enfermedad puede contribuir al aumento de la misma [23].

Si tenemos en cuenta que en el presente estudio prevalecen los sujetos con más de 5 años de evolución, es de esperar que este grupo de pacientes se caracterice por un alto índice de discapacidad.

La discapacidad tiene un profundo impacto en el bienestar emocional y la calidad de vida del paciente con dolor crónico, al interferir en todos los ámbitos de la vida como el autocuidado, las relaciones sociales, las funciones laborales y del hogar, el ocio y la recreación, además de la 
carga socioeconómica que representa por conceptos de tratamiento médicos y ausentismo laboral [23].

Lo anterior se refuerza al analizar las actividades de la vida cotidiana que más se ven afectadas por la discapacidad, donde predomina la imposibilidad de levantar pesos, estar de pie o sentado, acciones necesarias para que un individuo mantenga su autonomía e independencia y para el desarrollo de cualquier labor.

Es necesario destacar que el grupo más afectado en cuanto a la discapacidad es el de mayores de 60 años, lo que puede tener una clara explicación a través de los cambios y transformaciones que ocurren en el organismo, unido a otros padecimientos crónicos y a estilos de vida sedentarios propios de esta etapa del desarrollo [24].

Por último, al relacionar la ocupación con los diagnósticos etiológicos se observa un claro predominio de la aparición de las patologías que provocan dolor crónico de la espalda en personas que realizan esfuerzo físico frecuente, aspectos ampliamente relacionados en la literatura científica $(12,16)$.

Se puede concluir diciendo que las personas en edades laboralmente activas se encuentran sujetas a una serie de factores físicos, propios de las actividades de la vida cotidiana, que por demás están matizadas por un enfoque de género que se deriva de la cultura y el proceso de socialización, resultando en este estudio que la mayor prevalencia de dolor crónico estuvo presente en el sexo femenino.

A pesar de haber recibido tratamiento médico para el alivio del dolor, en la mayoría de los pacientes no se evidenciaron importantes mejorías clínicas, reflejándose el dolor con relativa intensidad y manteniéndose por un tiempo prolongado, lo que pudiera estar asociado a la no contemplación de los factores subjetivos.

\section{BIBLIOGRAFÍA}

1. Rull M, Miralles RC, Miralles I. Dolor de espalda. Diagnóstico. Enfoque general del tratamiento. Disponible en: http://www. scartd.org/arxius/lumbalgia-rull05.pdf.

2. Pérez Martín Y, Pérez Muñoz M. Los factores psicosociales en el dolor crónico. Intervención fisioterapéutica desde un enfoque biopsicosocial. RIECS. 2018;3(1):39-53. DOI: 10.37536/RIECS.2018.3.1.71.

3. Görge M, Ziehm J, Farin E. Health-care utilization of patients with chronic back pain before and after rehabilitation. BMC Health Serv Res. 2017;17(1):812. DOI: 10.1186/s12913017-2757-3.pdf.

4. Singh G, Newton C, O'Sullivan K, Soundy A, Heneghan NR. Exploring the lived experience and chronic low back pain beliefs of English-speaking Punjabi and white British people: a qualitative study within the NHS. BMJ Open. 2018;8(2):e020108. DOI: 10.1136/bmjopen-2017-020108.

5. GBD 2015 Disease and Injury Incidence and Prevalence Collaborators. Global, regional, and national incidence, prevalence, and years lived with disability for 310 diseases and injuries, 1990-2015: a systematic analysis for the Global Burden of Disease Study 2015. Lancet. 2016;388:1545602. DOI: 10.1016/S0140-6736(16)31678-6.

6. Santiago Bazàn C, Pérez Domingue KJ, Castro Reyes NL. Dolor lumbar y su relación con el índice de discapacidad en un Hospital de rehabilitación. Rev Cient Cienc Med. 2018;21(2):13-20.

7. Informe anual del Sistema Nacional de Salud 2017. Resumen ejecutivo. Madrid: Ministerio de Sanidad, Consumo y Bienestar Social. Centro ed publicaciones; 2017. Disponible en: https://www.mscbs.gob.es/estadEstudios/estadisti-
cas/sislnfSanSNS/tablasEstadisticas/InfAnualSNS2017/ ResumenEjecutivo2017.pdf.

8. Pomares A, Rodríguez T, Pomares J. Variables psicológicas y clínicas presentes en pacientes portadores de dolor crónico de espalda. Revista Finlay. 2016;6(1):49-58.

9. Ramos Y, Santana AR, Valladares AM, López L, González M. Relación entre estados emocionales y variables clínicas en pacientes con dolor crónico lumbar. Rev Cubana Med Gen Integr. 2017;33(2):180-90.

10. Pomares Avalos AJ, López Fernández R, Zaldívar Pérez DF. Validación de la escala de incapacidad por dolor lumbar de Oswestry, en paciente con dolor crónico de la espalda. Cienfuegos, 2017-2018. Rehabilitación (Madr). 2020;54(1):2530. DOI: 10.1016/j.rh.2019.10.003.

11. Espinosa García MV, Prieto Checa I. Mitos y realidades en torno al tratamiento del dolor crónico no oncológico. ¿Son los ancianos diferentes? Aten Primaria. 2019;51(8):514. DOI: 10.1016\%2Fj.aprim.2019.02.007.

12. Kao PY, Chan D, Samartzis D, Sham PC, Song YQ. Genetics of lumbar disk degeneration: technology, study designs, and risk factors. Orthop Clin North Am. 2011;42(4):479-86. DOI: 10.1016/j.ocl.2011.07.011.

13. Carneiro de Araújo $\mathrm{C}$, Ashmawi $\mathrm{H}$, Posso I. Sexo y percepción del dolor y analgesia. Rev Bras Anestesiol. 2011;61(6):449-58.

14. Gutiérrez W, Gutiérrez S. Diferencias de sexo en el dolor. Una aproximación a la clínica. Rev Colomb Anestesiol. 2012;40(3):207-12. DOI: 10.1016/j.rca.2012.05.007.

15. Guibert Reyes W, Prendes Labrada M, González Pérez R, Valdés Pérez EM. Influencia en la salud del rol de género. Rev Cubana Med Gen Integr. 1999;15(1).

16. Sánchez Zambrano C, del Roció Samaniego, Pesantez Piedra MD, Gran Benites ME. Dolor de espalda baja (Lumbalgia), enfermedad que no discrimina: Clasificación, Diagnóstico y tratamiento. Recimundo. 2019;3(2):610-27.

17. Bello-Villanueva A, Benítez-Lara M, Oviedo-Trespalacios 0. Características del dolor, aspectos psicológicos, calidad de vida y estrategias de afrontamiento en pacientes con dolor de espalda crónico en una ciudad de Colombia. Rev Colomb Anestesiol. 2017;45(4):310.6. DOI: 10.1016/j.rca.2017.07.002.

18. Breivik H, Collett B, Ventafridda V, Cohen R, GallacherD. Survey of chronic pain in Europe: prevalence, impact on daily life, and treatment. Eur J Pain. 2006;10(4):287-333. DOI: 10.1016/j.ejpain.2005.06.009.

19. Badia X, Fernández E, Segura A. Influence of sociodemographic and health status variables on evaluation of health states in a Spanish population. Eur J Public Health. 1995;5(21):8793. DOI: 10.1093/eurpub/5.2.87.

20. Fordyce WE, Brockway JA, Bergman JA, Spengler D. Acute Back Pain: A Control-Group Comparison of Behavioral vs Traditional Management Methods. J Behav Med. 1986;9(2):127-40. DOI: 10.1007/BFO0848473.

21. Díaz-Cerrillo JL, Rondón-Ramos A, Clavero-Cano S, Pérez-González R, Martinez-Calderon J, Alejandro Luque-Suarez A. Valoración del cumplimiento de un programa de escuela de espalda vs. el tratamiento farmacológico en la lumbalgia crónica. Aten Primaria. 2019;51(1):3-10. DOl: 10.1016/j.aprim.2017.07.003.

22. Boris Araos S. Manejo Multidisciplinario del dolor crónico. Rev Med Clin Condes. 2007;18(3):222-8.

23. Castro Reyes NL, Domínguez Pérez JK. Cronicidad del dolor lumbar y su relación con el grado de discapacidad física en los pacientes que asisten al hospital de rehabilitación del Callao. 2018.Disponible en: http://repositorio.ucss.edu.pe/handle/UCSS/715.

24. Cerquera A, Uribe A, Matajira Y, Correa H. Dependencia funcional y dolor crónico asociados a la calidad de vida del adulto mayor. Psicogente. 2017;20(38):398-409. DOI: 10.17081/psico.20.38.2561 